\title{
Isometric composition operators acting on the Chebyshev space
}

\author{
Thomas E. Goebeler, Jr. and Ashley L. Potter \\ (Communicated by David Larson)
}

\begin{abstract}
Norms of certain composition operators are given in terms of their symbols in some finite-dimensional setting. Then a family of isometric composition operators acting on certain vector spaces is identified.
\end{abstract}

\section{Introduction}

Much research has been done concerning composition operators over the last five decades. However, this research has primarily focused on a host of standard questions about composition operators in the realm of complex functions. Recently the first author has proposed a number of questions regarding composition operators in the real function area for undergraduate student research supported by the Ursinus College Summer Fellows Program [Doperak 2006; Gareau 2005; Kunaszuk 2006; Potter 2007]. This topic offers accessibility for undergraduate research while providing fertile ground for genuinely new results.

This paper focuses on one particular real function space, the Chebyshev space, $T$, where we explore norm-related ideas for composition operators, specifically norms and isometries. The ultimate goal of this research is to find the norm of a composition operator $C_{g}$ acting on the Chebyshev space in terms of its symbol $g$. We begin by considering norms of composition operators in the infinitedimensional space, and move on to examine the topic in the finite-dimensional space. After looking at the various finite-dimensional subspaces, we begin to look at qualities that would lead to the symbol inducing an isometry.

\section{Definitions}

We set down some terminology and basic facts here. A composition operator $C_{g}$ acts on functions $f$ according to the rule $C_{g}(f)=f \circ g$. The function $g$ is called the symbol of the operator. Necessarily $g$ must have range contained in the domain of

MSC2000: 47B33, 47B38.

Keywords: composition operator, norm, isometry. 
$f$ for this to make sense. The typical assumptions are that $C_{g}$ has a vector space $\mathscr{F}_{F}$ of functions as its domain, that the functions $f \in \mathscr{F}$ and $g$ have a common domain $D$, and that $g(D) \subseteq D$.

To speak of an operator's norm, it must first be known that the operator is bounded on its domain space. The following definition is operational for any linear operator, not just composition operators.

Definition 1. A linear operator $A$ is bounded from a vector space $V$ to another vector space $W$ provided there exists an $M \in \mathbb{R}$ such that $\|A f\|_{W} \leq M \cdot\|f\|_{V}$ for all $f \in V$. This constant $M$ in the inequality is referred to as a bound.

If an operator $A: \mathscr{F} \rightarrow \mathscr{G}$ is known to be bounded, we can give it a norm (the operator norm), defined as $\|A\|=\sup _{f \neq 0}\|A f\|_{\varphi} /\|f\|_{\mathscr{F}}$.

The setting for the present work is a real function space. This is atypical of composition operator research, which sees the majority of work done on complex function spaces. We find this setting surprisingly rich, once we make some modifications to the questions we ask. The reader interested in more information regarding composition operators on spaces of complex functions can consult [Cowen and MacCluer 1995], which is widely regarded as the best resource for beginners in the field.

For the purposes of this paper, we make the following real-function definition of the Chebyshev space.

Definition 2. The Chebyshev space $T$ is herein the completion of the set of all continuous functions defined on the interval $[-1,1]$, taking on real values, and obeying the following integral convergence condition:

$$
\|f\|^{2}=\int_{-1}^{1}|f(x)|^{2} \frac{1}{\sqrt{1-x^{2}}} d x<\infty .
$$

There are many functions in the vector space $T$, including polynomials and many other elementary functions. In fact, the monomials form a basis for $T$.

Orthogonalizing the basis vectors $\left\{1, x, x^{2}, \ldots\right\}$ with respect to the inner product

$$
\langle u, v\rangle=\int_{-1}^{1} u(x) \overline{v(x)} \frac{1}{\sqrt{1-x^{2}}} d x
$$

leads to $1, x, x^{2}-\frac{1}{2}, \ldots$, which are the Chebyshev polynomials (of the first kind). However, the polynomials are most commonly normalized so that if $v_{n}$ is the $n$-th Chebyshev polynomial, $v_{n}(1)=1$. Doing so shows the first four are $v_{0}=1, v_{1}=$ $x, v_{2}=2 x^{2}-1, v_{3}=4 x^{3}-3 x$.

More information about the Chebyshev polynomials can be found in [Lebedev 1972]. Initially we seek a formula for the norm of $C_{g}$ in terms of a calculation involving the symbol $g$, but as it will be shown, it seems likely that $C_{g}$ is unbounded 
on the Chebyshev space for all but a few symbols. We thus reconsider the question of norm for the restriction of the composition operator to finite-dimensional subspaces.

Definition 3. The finite dimensional subspace $T_{n}$ of $T$ is the subspace of $T$ that contains all polynomials of degree at most $n$. More concisely, we can say that $T_{n}=\operatorname{Span}\left\{1, x, x^{2}, \ldots, x^{n}\right\}$.

To say $f \in T_{n}$ means that $f$ is a linear combination of the basis vectors, in other words, $f=c_{0} \cdot 1+c_{1} \cdot x+c_{2} \cdot x^{2}+\cdots+c_{n} \cdot x^{n}$.

Since $T_{n}$ is finite-dimensional we know that $C_{g}$ is automatically bounded, simply by virtue of being linear. See [Horn and Johnson 1985]. This turns out to be a crucial restriction that leads to both norm formulas and identification of isometric composition operators. For more information regarding norms, see [Akhiezer and Glazman 1993; Dunford and Schwartz 1958; Reed and Simon 1980].

\section{Preliminary investigation}

As is always the case when the domain and range spaces are the same and use the same norm, the identity function $g(x)=x$ induces a composition operator with norm 1:

$$
\left\|C_{g}\right\|=\sup _{f \neq 0} \frac{\left\|C_{g}(f)\right\|}{\|f\|}=\frac{\|f \circ g\|}{\|f\|}=\frac{\|f\|}{\|f\|}=1 .
$$

The next example suggests most composition operators will fail to be bounded on $T$. Consider the symbol $g(x)=a x$ for $|a|<1$. In the following, suppose $0<a<1$. This condition is imposed to guarantee that range $(g) \subset[-1,1]$. A straightforward substitution (valid since $g$ is an increasing absolutely continuous function) leads us to

$$
\begin{aligned}
\left\|C_{a x}(f)\right\|^{2} & =\int_{-1}^{1}\left|C_{a x}(f)(x)\right|^{2} \frac{1}{\sqrt{1-x^{2}}} d x=\int_{-1}^{1}[f(a x)]^{2} \frac{1}{\sqrt{1-x^{2}}} d x \\
& =\frac{1}{a} \int_{-a}^{a}[f(u)]^{2} \frac{1}{\sqrt{1-\left(\frac{u}{a}\right)^{2}}} d x .
\end{aligned}
$$

But $\frac{u}{a} \geq u$ for $a \in(0,1)$ so

$$
\frac{1}{\sqrt{1-\left(\frac{u}{a}\right)^{2}}} \geq \frac{1}{\sqrt{1-u^{2}}} .
$$

Thus,

$$
\left\|C_{a x}(f)\right\|^{2} \geq \frac{1}{a} \int_{-a}^{a}[f(u)]^{2} \frac{1}{\sqrt{1-u^{2}}} d u .
$$


If this lower bound is not convergent as an improper integral, the operator is unbounded; therefore this case will be investigated further. Let

$$
f_{a}(x)=\frac{1}{\sqrt[4]{|a-x|}}
$$

and observe that $f_{a} \in T$, as

$$
\begin{aligned}
\int_{-1}^{1}\left|f_{a}\right|^{2} \frac{1}{\sqrt{1-x^{2}}} d x & =\int_{-1}^{1}\left|\frac{1}{\sqrt[4]{|a-x|}}\right|^{2} \frac{1}{\sqrt{1-x^{2}}} d x \\
& =\int_{-1}^{1} \frac{1}{\sqrt{1-x}} \frac{1}{\sqrt{1+x}} \frac{1}{\sqrt{|a-x|}} d x<\infty
\end{aligned}
$$

is an improper integral with singularities as both endpoints of the interval of integration and at $x=a$. Note $\left(C_{a x}\left(f_{a}\right)\right)(x)=f_{a}(a x)=|a|^{-1 / 4}|1-x|^{-1 / 4}$. Therefore,

$$
\begin{aligned}
& \left\|C_{a x}\left(f_{a}\right)\right\|^{2}=\int_{-1}^{1}\left|f_{a}(a x)\right|^{2} \frac{1}{\sqrt{1-x^{2}}} d x=\int_{-1}^{1}\left|\frac{1}{\sqrt[4]{|a|}} \cdot \frac{1}{\sqrt[4]{1-x}}\right|^{2} \frac{1}{\sqrt{1-x^{2}}} d x \\
& =\frac{1}{\sqrt{|a|}} \int_{-1}^{1} \frac{1}{\sqrt{1-x}} \cdot \frac{1}{\sqrt{1-x}} \cdot \frac{1}{\sqrt{1+x}} d x=\frac{1}{\sqrt{|a|}} \int_{-1}^{1} \frac{1}{1-x} \cdot \frac{1}{\sqrt{1+x}} d x=\infty .
\end{aligned}
$$

The divergence is driven by the factor $1 /(1-x)$. We conclude that $C_{a x}$ is unbounded for $a \in(0,1)$, and, by symmetry, for $a \in(-1,0)$. Note that the operator $C_{-x}$ is bounded since $C_{x}$ is bounded and the integral and weight are symmetric about 0 . (For the reader with a background in Lebesgue spaces, notice also that when $a=0, C_{a x}$ amounts to being the operator of point-evaluation at 0 . Since our space is really the space $L^{2}\left([-1,1], d x / \sqrt{1-x^{2}}\right)$, that is, the completion of the polynomials in the Chebyshev norm, $C_{0}$ fails to make sense. Indeed, all point-evaluation operators on such an $L^{2}$-space are unbounded.)

A similar argument shows that when $g(x)=a x+b$, with range $(g) \subseteq[-1,1]$, $C_{g}$ is again unbounded. Algebra shows the conditions on $a$ and $b$ are $|a| \leq 1$ and $|b| \leq 1-|a|$. This means $\operatorname{graph}(g) \subseteq[-1,1] \times[-1,1]$; this Cartesian product of intervals will be called the "box." We include some examples to illustrate the phrases "in the box" and "out of the box" (Figure 1).

Again, a test function shows $C_{g}$ is unbounded: let $f_{a, b}(x)=1 / \sqrt[4]{|a+b-x|}$ and perform the norm calculation of $C_{a x+b}\left(f_{a, b}\right)$.

\section{A change of venue}

The unboundedness of operators with such elementary symbols leads us to restrict the operator $C_{g}$ to finite-dimensional subspaces of $T$. 

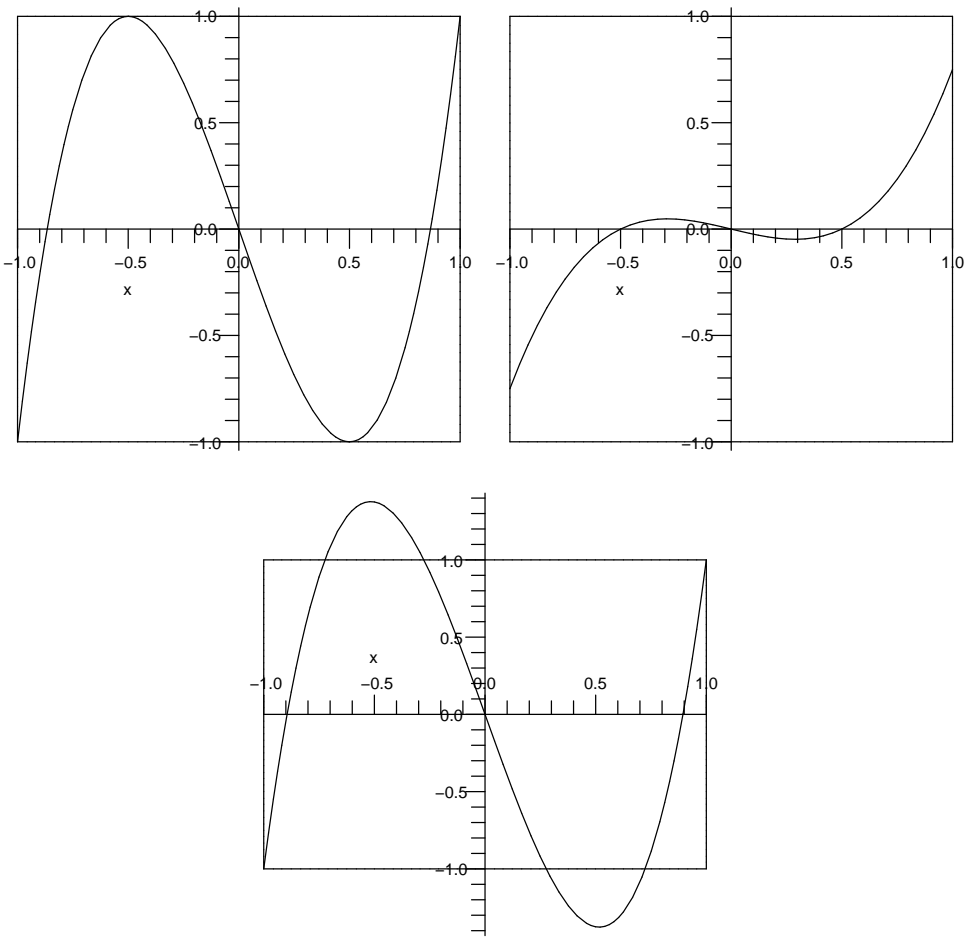

Figure 1. $g(x)$ in the box (top line); $g(x)$ outside the box (bottom).

4.1. $\boldsymbol{T}_{\mathbf{1}} \rightarrow \boldsymbol{T}_{\mathbf{1}}$. In order for $C_{g}$ to map $T_{n}$ back to $T_{n}, g$ must be at most degree one and thus $g(x)=a x+b$ for some $a, b \in \mathbb{R}$. A concrete subcase would be $T_{1} \rightarrow T_{1}$, and thus input functions are of the form $c_{0}+c_{1} x$ while $g(x)=a x+b$. In order for $C_{g}: T_{n} \rightarrow T_{2 n}, g$ must be at most degree two, $g(x)=a x^{2}+b x+c$ for $a, b, c \in \mathbb{R}$. A concrete subcase would be $T_{1} \rightarrow T_{2}$, and thus again $f \in T_{1}$ has the form $c_{0}+c_{1} x$ while $g(x)=a x^{2}+b x+c$.

Employing the definition of the norm of an operator we have

$$
\begin{aligned}
\left\|C_{g}\right\|_{T_{1} \rightarrow T_{1}}^{2} & =\sup _{f \neq 0} \frac{\left\|C_{g}(f)\right\|^{2}}{\|f\|^{2}}=\max _{f \neq 0} \frac{\left\|C_{g}(f)\right\|^{2}}{\|f\|^{2}} \\
& =\max _{c_{0}^{2}+c_{1}^{2} \neq 0} \frac{\int_{-1}^{1}\left(c_{0}+c_{1}(a x+b)\right)^{2} \frac{1}{\sqrt{1-x^{2}}} d x}{\int_{-1}^{1}\left(c_{0}+c_{1} x\right)^{2} \frac{1}{\sqrt{1-x^{2}}} d x} \\
& =\max _{c_{0}^{2}+c_{1}^{2} \neq 0} \frac{a^{2} c_{1}^{2}+2 b^{2} c_{1}^{2}+4 b c_{1} c_{0}+2 c_{0}^{2}}{c_{1}^{2}+2 c_{0}^{2}} .
\end{aligned}
$$

It is proper to replace the supremum with a maximum since we are working on a finite dimensional space; details of this thought can be found in [Horn and 

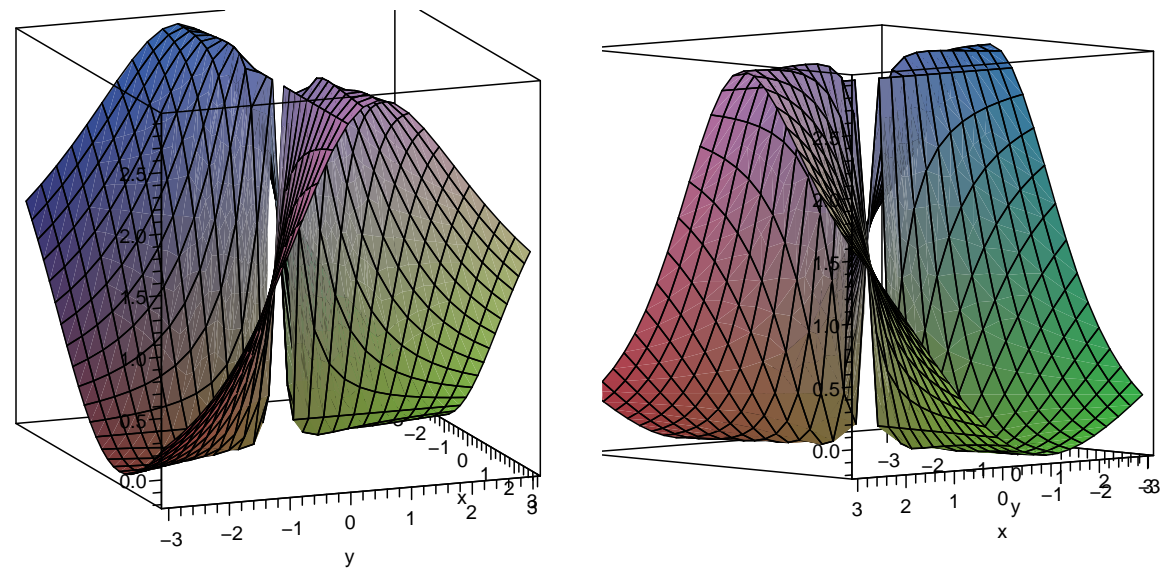

Figure 2. Two views of the "ridge".

Johnson 1985]. The quantity being maximized in the last line will be called the norm quotient.

After finding partial derivatives, and solving for $c_{1}$, critical lines are found. These critical lines correspond to "ridges" of the norm quotient, see Figure 2, rather than isolated maxima or minima.

The following critical lines were found:

$$
c_{1}=\frac{c_{0}\left(a^{2}+2 b^{2}-1\right) \pm c_{0} \sqrt{\left(a^{2}+2 b^{2}-1\right)^{2}+8 b^{2}}}{2 b} .
$$

After expanding and simplifying, the following formula is obtained:

$$
\left\|C_{g}\right\|_{T_{1} \rightarrow T_{1}}^{2}=\frac{\sqrt{\left(a^{2}+2 b^{2}-1\right)^{2}+8 b^{2}}+a^{2}+2 b^{2}+1}{2} .
$$

At this point an interesting question arises: how do these norms change when viewing the operators successively $T_{2} \rightarrow T_{2}, T_{3} \rightarrow T_{3}, \ldots, T_{n} \rightarrow T_{n}, \ldots$, as $n \rightarrow \infty$ ? In other words, can we determine for all symbols $g$ the limit $\lim _{n \rightarrow \infty}\left\|C_{g}\right\|_{T_{n} \rightarrow T_{n}}$ ?

4.2. $\boldsymbol{T}_{\mathbf{1}} \rightarrow \boldsymbol{T}_{\mathbf{2}}$. We restrict the domain of $C_{g}$ to polynomials of the form $c_{0}+c_{1} x$ and restrict the form of the symbol to $a x^{2}+b x+c$. Thus we are viewing the operator as $C_{g}: T_{1} \rightarrow T_{2}$. Throughout this section, we assume $a \neq 0$, or else this reduces to the situation in Section 4.1, $T_{1} \rightarrow T_{1}$.

Before proceeding with the calculation of the norm, we will address the constraints on $a, b$, and $c$ for $g(x)$ to stay within the $[-1,1]$ interval. By inspection, $|c| \leq 1$ because otherwise $g(x)$ would be outside the box, for then it would have an intercept outside the box. Using the fact that $|c| \leq 1$, it becomes clear that $|a+c| \leq 1$. To see this, consider the endpoints. Evaluation of $g$ at -1 yields 
$-1 \leq a-b+c \leq 1$. Evaluation of $g$ at 1 yields $-1 \leq a+b+c \leq 1$. Adding these inequalities gives us $-2 \leq 2 a+2 c \leq 2$ which can be reduced to $-1 \leq a+c \leq 1$ and thus $|a+c| \leq 1$.

By combining the previous constraints, it must be true that $|a| \leq 2$. To put constraints on $b$, in terms of $a$ and $c$, there are two conditions:

- The values of $a x^{2}+b x+c$ at the endpoints of the interval $[-1,1]$ cannot be above or below the corners of the $[-1,1] \times[-1,1]$ box.

- If the vertex of $a x^{2}+b x+c$ occurs for $x \in[-1,1]$, it cannot be above the upper boundary or below the lower boundary of the $[-1,1] \times[-1,1]$ box.

The endpoints concerning $b$ will be addressed first:

$$
\begin{aligned}
g(-1) & =a-b+c, & g(1) & =a+b+c, \\
-1 & \leq a-b+c \leq 1, & -1 & \leq a+b+c \leq 1, \\
-1-a-c & \leq-b \leq 1-a-c, & -1-a-c & \leq b \leq 1-a-c, \\
-1+(a+c) & \leq b \leq 1+(a+c), & -1-(a+c) & \leq b \leq 1-(a+c) .
\end{aligned}
$$

For $a+c>0$, it is clear that

$$
-1-(a+c) \leq-1+(a+c) \leq b \leq 1-(a+c) \leq 1+(a+c) .
$$

Thus, $-1+(a+c) \leq b \leq 1-(a+c)$, or, $|b| \leq|1-(a+c)|$. For $a+c<0$, following a similar approach, we arrive at $|b| \leq|1+(a+c)|$. Combining both cases, the inequality limiting the values of $b$ is

$$
|b| \leq|1-| a+c||
$$

The next condition for the bounds of $b$ concerns the vertex location within the $[-1,1] \times[-1,1]$ box. The vertex is where $x=-\frac{b}{2 a}$. (Recall that we have assumed $a \neq 0$.) If the vertex is outside the box $(|b|>2|a|)$, we need only consider the values of the symbol at $x= \pm 1$, as above. However, if the vertex is in the box $(|b| \leq 2|a|)$, further constraints must be imposed for $\operatorname{graph}(g)$ to be in the box.

To find the bounds on $b$ when the vertex is inside the box, calculate:

$$
g\left(-\frac{b}{2 a}\right)=a\left(-\frac{b}{2 a}\right)^{2}+b\left(-\frac{b}{2 a}\right)+c .
$$

We require $\left|a(-b / 2 a)^{2}+b(-b / 2 a)+c\right| \leq 1$, which simplifies to $\left|c-b^{2} / 4 a\right| \leq 1$. Algebra shows we therefore want $-4(1-c) \leq b^{2} / a \leq 4(1+c)$.

This leads to two cases, depending upon the sign of $a$. If $a>0$, the necessary condition is $-4 a(1-c) \leq b^{2} \leq 4 a(1+c)$, or, $|b| \leq 2 \sqrt{a(1+c)}$. If $a<0$, the necessary condition is $4 a(1+c) \leq b^{2} \leq-4 a(1-c)$, or, $|b| \leq 2 \sqrt{-a(1-c)}$. 
Summarizing, for graph $(g)$ to be in the box, we need

$$
\left\{\begin{array}{l}
|c| \leq 1 \\
|a+c| \leq 1 \\
|b| \leq|1-| a+c||
\end{array}\right.
$$

and if $|b| \leq 2|a|$, also

$$
\begin{cases}|b| \leq \sqrt{a(1+c)}, & a>0 \\ |b| \leq \sqrt{-a(1-c)}, & a<0\end{cases}
$$

Now that the constraints on $a, b$, and $c$ for $g(x)=a x^{2}+b x+c$ to stay within the $[-1,1] \times[-1,1]$ box have been established, computing the actual norm of the composition operator will be the next step.

With $0 \neq f \in T_{1}$,

$$
\begin{aligned}
\left\|C_{g}\right\|_{T_{1} \rightarrow T_{2}}^{2} & =\sup \frac{\left\|C_{g}(f)\right\|^{2}}{\|f\|^{2}}=\max \frac{\int_{-1}^{1}\left[c_{0}+c_{1}\left(a x^{2}+b x+c\right)\right]^{2} \frac{1}{\sqrt{1-x^{2}}} d x}{\int_{-1}^{1}\left[c_{0}+x_{1} x\right]^{2} \frac{1}{\sqrt{1-x^{2}}} d x} \\
& =\max \frac{3 a^{2} c_{1}^{2}+8 a c_{1}\left(c c_{1}+c_{0}\right)+4\left(b^{2} c_{1}+2\left(c^{2} c_{1}^{2}+2 c c_{0} c_{1}+c_{0}^{2}\right)\right)}{4\left(2 c_{0}^{2}+c_{1}^{2}\right)} .
\end{aligned}
$$

The quantity being maximized in the last line will be called the norm quotient. After finding partial derivatives the following critical points are found:

$c_{1}=\frac{c_{0}\left(3 a^{4}+8 a c+4 b^{2}+8 c-4\right)}{4 a+8 c}$

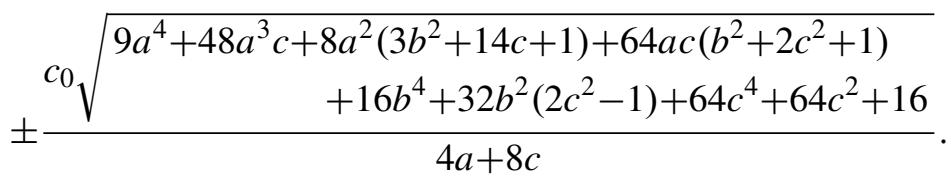

To attain a formula, the value for $c_{1}$ is substituted into the norm quotient. After expanding and simplifying using a computer algebra system, the following formula is obtained:

$$
\left\|C_{g}\right\|_{T_{1} \rightarrow T_{2}}^{2}=\frac{\sqrt{\begin{array}{c}
9 a^{4}+48 a^{3} c+8 a^{2}\left(3 b^{2}+14 c+1\right)+64 a c\left(b^{2}+2 c^{2}+1\right) \\
+16 b^{4}+32 b^{2}\left(2 c^{2}-1\right)+64 c^{4}+64 c^{2}+16
\end{array}}}{8 \quad+\frac{b^{2}}{2}+c^{2}+a c+\frac{3 a^{2}+4}{8} .}
$$

We can raise the companion question as to the norm of the operator when viewing it successively $T_{2} \rightarrow T_{4}, T_{3} \rightarrow T_{6}, \ldots, T_{n} \rightarrow T_{2 n}, \ldots$, as $n \rightarrow \infty$. 
4.3. $\boldsymbol{T}_{\mathbf{1}} \rightarrow \boldsymbol{T}_{\mathbf{3}}$. Following the template of the previous section, we discover the norm of a composition operator in the $T_{1} \rightarrow T_{3}$ subspace:

$$
\begin{aligned}
\left\|C_{g}\right\|_{T_{1} \rightarrow T_{3}}^{2}= & \frac{5 a^{2}+12 a c+2\left(3 b^{2}+8 b d+4\left(c^{2}+2 d^{2}+1\right)\right)}{16} \\
+\frac{1}{16}( & 25 a^{4}+120 a^{3} c+4 a\left(15 b^{2}+40 b d+4\left(14 c^{2}+5\left(2 d^{2}-1\right)\right)\right) \\
& +48 a c\left(3 b^{2}+8 b d+4\left(c^{2}+2 d^{2}-1\right)\right) \\
+ & 4\left(9 b^{4}+48 b^{3} d+8 b^{2}\left(3 c^{2}+14 d^{2}+1\right)+64 b d\left(c^{2}+2 d^{2}+1\right)\right) \\
& \left.+64\left(c^{4}+2 c^{2}\left(2 d^{2}-1\right)+4 d^{4}+4 d^{2}+1\right)\right)^{1 / 2} .
\end{aligned}
$$

Likewise we can ask about norms viewing the operator $T_{2} \rightarrow T_{6}, T_{3} \rightarrow T_{9}, \ldots$, $T_{n} \rightarrow T_{3 n}, \ldots$, as $n \rightarrow \infty$.

There are more potentially interesting questions open to us as the number of coefficients increases. Finding a norm formula for general symbols is the ultimate goal.

\section{Isometries}

Definition 4. An isometry is a bijective map $f: X \rightarrow Y$ between two normed spaces that preserves lengths, that is, $\|f(x)\|_{Y}=\|x\|_{X}$, where $\|\cdot\|_{X}$ and $\|\cdot\|_{Y}$ are the norms associated with the spaces $X$ and $Y$.

For our purposes, the isometry will be viewed as acting between two finitedimensional subspaces $T_{n}$ and $T_{m}$ of $T$. More precisely, $C_{g}$ will act as an isometry when the norm of the input $\|f\|$ equals the norm of the output $\left\|C_{g}(f)\right\|$, and thus automatically $\left\|C_{g}\right\|=1$.

Theorem 5. When the symbol $g(x)$ is a normalized Chebyshev polynomial in the subspace $T_{n}, n>0$, the induced operator $C_{g}: T_{1} \rightarrow T_{n}$ is an isometry.

Proof. Let $v_{n}(x)$ be the Chebyshev polynomial (so the symbol $g(x)$ is $v_{n}(x)$, the polynomial of degree $n$ ) and $f(x)$ any nonzero linear polynomial. Then

$$
\begin{aligned}
& \left\|C_{v_{n}}\right\|^{2}=\sup \frac{\left\|C_{v_{n}}(f)\right\|^{2}}{\|f\|^{2}}=\max \frac{\left\|f \circ v_{n}\right\|^{2}}{\|f\|^{2}}=\max \frac{\int_{-1}^{1}\left[c_{0}+c_{1}\left(v_{n}(x)\right)\right]^{2} \frac{1}{\sqrt{1-x^{2}}} d x}{\int_{-1}^{1}\left[c_{0}+c_{1} x\right]^{2} \frac{1}{\sqrt{1-x^{2}}} d x} \\
& =\max \frac{\int_{-1}^{1}\left[c_{0}^{2}+2 c_{0} c_{1} v_{n}(x)+c_{1}^{2}\left(v_{n}(x)\right)^{2}\right] \frac{1}{\sqrt{1-x^{2}}} d x}{\int_{-1}^{1}\left[c_{0}^{2}+2 c_{0} c_{1} x+c_{1}^{2} x^{2}\right] \frac{1}{\sqrt{1-x^{2}}} d x} \\
& =\max \frac{\int_{-1}^{1} c_{0}^{2} \frac{1}{\sqrt{1-x^{2}}} d x+2 c_{0} c_{1} \int_{-1}^{1} v_{n}(x) \frac{1}{\sqrt{1-x^{2}}} d x+c_{1}^{2} \int_{-1}^{1}\left(v_{n}(x)\right)^{2} \frac{1}{\sqrt{1-x^{2}}} d x}{\frac{\pi}{2}\left[c_{1}^{2}+2 c_{0}^{2}\right]} .
\end{aligned}
$$




$$
\begin{aligned}
\text { But } \int_{-1}^{1} v_{n}(x) \frac{1}{\sqrt{1-x^{2}}} d x=0 \text { when } 0 \neq n \text {. So, } \\
\qquad C_{v_{n}} \|^{2}=\max \frac{\pi c_{0}^{2}+\frac{1}{2} \pi c_{1}^{2}}{\frac{1}{2} \pi\left(c_{1}^{2}+2 c_{0}^{2}\right)}=1 .
\end{aligned}
$$

This calculation shows that the norm quotient is independent of the choice of $c_{0}$ and $c_{1}$, and thus $\left\|C_{v_{n}}(f)\right\|=\|f\|$.

We were encouraged to look for more isometric composition operators and began with those acting from $T_{1} \rightarrow T_{3}$. With the previous theorem in mind, the first general form of the symbol considered was $g(x)=a x^{3}+c x$, since that is the form of the Chebyshev polynomial of degree three. With $0 \neq f \in T_{1}$,

$$
\begin{aligned}
\frac{\left\|C_{a x^{3}+c x}(f)\right\|^{2}}{\|f\|^{2}} & =\frac{\left\|f \circ\left(a x^{3}+c x\right)\right\|^{2}}{\|f\|^{2}}=\frac{\int_{-1}^{1}\left[c_{0}+c_{1}\left(a x^{3}+c x\right)\right]^{2} \frac{1}{\sqrt{1-x^{2}}} d x}{\int_{-1}^{1}\left[c_{0}+c_{1} x\right]^{2} \frac{1}{\sqrt{1-x^{2}}} d x} \\
& =\frac{5 a^{2} c_{1}^{2}+12 a c c_{1}^{2}+8\left(c^{2} c_{1}^{2}+2 c_{0}^{2}\right)}{8\left(c_{1}^{2}+2 c_{0}^{2}\right)} .
\end{aligned}
$$

To find what the values of $a$ and $c$ must be for $C_{g}$ to act as an isometry, we forced this norm quotient to be 1 . This leads to $25 a^{2}+40 c^{2}=40$, the equation of an ellipse, which amounts to requiring

$$
c=\frac{-3 a \pm \sqrt{16-a^{2}}}{4} .
$$

We can conclude that any symbol of form $g(x)=a x^{3}+c x$, with $c$ as in (5-1), will act as an isometry in $T_{1} \rightarrow T_{3}$, assuming the symbol is admissible.

We continued this technique with the symbols $g(x)=a x^{3}+d, g(x)=a x^{3}+$ $b x^{2}+c x, g(x)=a x^{3}+c x+d$, and $g(x)=a x^{3}+b x^{2}+d$, as well as other examples. However, each time we found dependence on $c_{0}$ and $c_{1}$, and thus $C_{g}$ could not act isometrically on the whole subspace.

With the success of finding the isometry family for the symbol $g(x)=a x^{3}+c x$ in comparison to the lack of success with the examples explored above, we wondered if this was the only form of $g$ that could act as an isometry. We backtracked to the general form $g(x)=a x^{3}+b x^{2}+c x+d$ and constructed some test functions.

From Section 4.3 where we found $\left\|C_{g}\right\|_{T_{1} \rightarrow T_{3}}$, we began testing with various functions $f \in T_{1}$ to find necessary conditions on the form of $g$ for $C_{g}$ to be an isometry. The first such functions are $f_{1}(x)=x+1$ and $f_{2}(x)=x-1$. Recall that

$$
\begin{aligned}
& \frac{\left\|C_{g}(f)\right\|^{2}}{\|f\|^{2}}= \\
& \quad \frac{5 a^{2} c_{1}^{2}+12 a c c_{1}^{2}+2\left(3 b^{2} c_{1}^{2}+8 b c_{1}\left(d c_{1}+c_{0}\right)+4\left(c^{2} c_{1}^{2}+2\left(d^{2} c_{1}^{2}+2 d c_{0} c_{1}+c_{0}^{2}\right)\right)\right)}{8\left(2 c_{0}^{2}+c_{1}^{2}\right)} .
\end{aligned}
$$


We see that

$$
\begin{aligned}
& \frac{\left\|C_{g}(x+1)\right\|_{T_{3}}^{2}}{\|x+1\|_{T_{1}}^{2}}=\frac{5 a^{2}+12 a c+2\left(3 b^{2}+8 b(d+1)+4\left(c^{2}+2\left(d^{2}+2 d+1\right)\right)\right)}{24}, \\
& \frac{\left\|C_{g}(x-1)\right\|_{T_{3}}^{2}}{\|x-1\|_{T_{1}}^{2}}=\frac{5 a^{2}+12 a c+2\left(3 b^{2}+8 b(d-1)+4\left(c^{2}+2\left(d^{2}-2 d+1\right)\right)\right)}{24} .
\end{aligned}
$$

For $C_{g}$ to qualify as an isometry, each norm quotient must be 1 and thus the difference of the two norm quotients must be 0 :

$$
0=\left\|C_{g}(x+1)\right\|_{T_{3}}^{2}-\left\|C_{g}(x+1)\right\|_{T_{3}}^{2}=\frac{4 b-8 d}{3} .
$$

Thus $\frac{4 b-8 d}{3}=0$, or $b=2 d$. Next we used $f_{3}(x)=x+\frac{1}{2}$, and $f_{4}(x)=x-\frac{1}{4}$. The norm quotients for $f_{3}(x)$ and $f_{4}(x)$ with $b=2 d$ are

$$
\begin{aligned}
& \frac{\left\|C_{g}\left(x+\frac{1}{2}\right)\right\|_{T_{3}}^{2}}{\left\|x+\frac{1}{2}\right\|_{T_{1}}^{2}}=\frac{5 a^{2}+12 a c+4\left(2 c^{2}+18 d^{2}+8 d+1\right)}{12}, \\
& \frac{\left\|C_{g}\left(x-\frac{1}{4}\right)\right\|_{T_{3}}^{2}}{\left\|x-\frac{1}{4}\right\|_{T_{1}}^{2}}=\frac{5 a^{2}+12 a c+8 c^{2}+72 d^{2}-16 d}{9} .
\end{aligned}
$$

We set each new norm quotient equal to 1 and solved for $d$. In solving

$$
\left\|C_{g}\left(x+\frac{1}{2}\right)\right\|_{T_{3}}^{2}=\left\|x+\frac{1}{2}\right\|_{T_{1}}^{2}
$$

for $d$, we find

$$
d_{1}^{ \pm}=-\frac{2}{9} \pm \frac{\sqrt{2} \sqrt{-45 a^{2}-4\left(27 a c+2\left(9 c^{2}-13\right)\right)}}{36} .
$$

While for $\left\|C_{g}\left(x-\frac{1}{4}\right)\right\|_{T_{3}}^{2}=\left\|x-\frac{1}{4}\right\|_{T_{1}}^{2}$ we have

$$
d_{2}^{ \pm}=\frac{1}{9} \pm \frac{\sqrt{2} \sqrt{-45 a^{2}-4\left(27 a c+2\left(9 c^{2}-10\right)\right)}}{36} .
$$

We are seeking coefficients for $g$ that will induce an isometry. Thus a single choice for $d$ must serve for all test functions. The expressions for $d$ above must therefore be equal and their difference 0 . We examined each of the four pairings and present the most illuminating one, which turns out to be $d_{1}^{+}=d_{2}^{-}$. Rearranging terms gives

$$
\frac{\sqrt{2} \sqrt{-45 a^{2}-4\left(27 a c+2\left(9 c^{2}-13\right)\right)}}{36}+\frac{\sqrt{2} \sqrt{-45 a^{2}-4\left(27 a c+2\left(9 c^{2}-10\right)\right)}}{36}=\frac{1}{3},
$$


which can be solved for $c$ by squaring both sides, isolating the product of radicals on one side of the equation, squaring again, and simplifying. The solution is

$$
c=\frac{-3 a \pm \sqrt{16-a^{2}}}{4}
$$

which is (5-1). When we substitute either expression for $c$ into (5-2), we find $d_{1}=0,-\frac{4}{9}$. Likewise, using (5-3), we get $d_{2}=0, \frac{2}{9}$.

Thus, for $C_{g}$ to be an isometry $d$ must be 0 , and since we already discovered $b=2 d$, necessarily $b=0$. Therefore for a symbol $g$ to induce an isometry is for $g$ to be of the form $g(x)=a x^{3}+c x$. This was the form first investigated at the start of this subsection where we discovered the only family of the form $g(x)=a x^{3}+c x$ is when $c$ is as in (5-1). Therefore, the only family that enables $g$ to induce as an isometry from $T_{1}$ to $T_{3}$ is the one described above.

Now that we have specified the form of the symbol $g$, we must determine the constraints on $g$ to be in the $[-1,1] \times[-1,1]$ box. That is, we must make sure there are symbols satisfying the condition for $C_{g}$ to be an isometry and which are themselves admissible.

There are two basic criteria for $g(x)=a x^{3}+c x$ to stay in the box:

- The values of $a x^{3}+c x$ at the endpoints of the interval $[-1,1]$ cannot be above or below the corners of the $[-1,1] \times[-1,1]$ box (that is, the graph enters the left side of the box and exits on the right, not the top or the bottom).

- If the local extrema of $a x^{3}+c x$ (if any) occur for $x \in[-1,1]$, they cannot be above the upper boundary or below the lower boundary of the $[-1,1] \times[-1,1]$ box (that is, the graph does not penetrate the top or bottom of the box).

The endpoints will be considered first; we require at the left endpoint $-1 \leq$ $-(a+c) \leq 1$, and at the right endpoint $-1 \leq a+c \leq 1$. These requirements can be summarized by $|a+c| \leq 1$. Graphically (with $a$ as the horizontal axis and $c$ as the vertical axis), this is represented by a "strip" in the $a c$ plane, between the lines $c=1-a$ and $c=-1-a$. This is seen in Figure 3 .

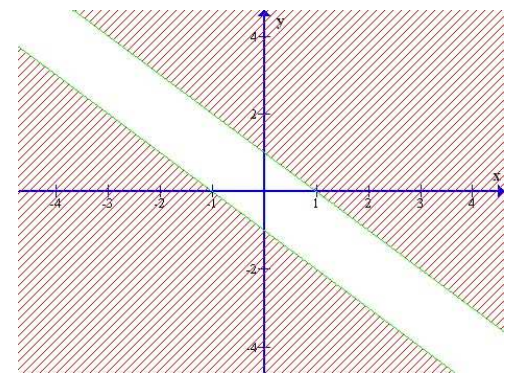

Figure 3. Strip of admissibility. The slanted shading indicates regions of inadmissibility. 

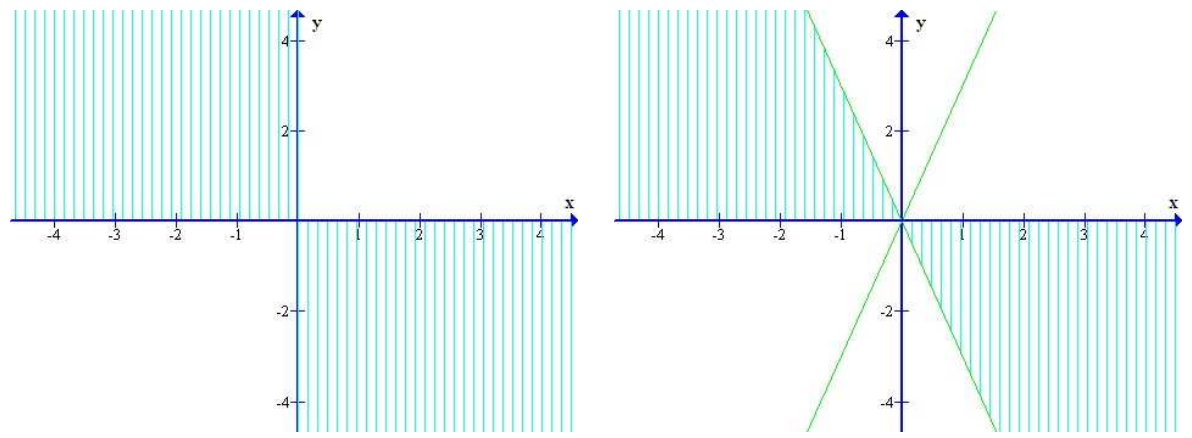

Figure 4. Regions of admissibility, when $a$ and $c$ have the same sign (left), and when $a$ and $c$ have opposite signs, $|c|>3|a|$ (right). The vertical shading indicates further investigation is needed.

Next, we consider the local extrema of $g(x)=a x^{3}+c x$ which occur when $x^{2}=-c / 3 a$. Two cases arise: when $a$ and $c$ have the same sign, and when $a$ and $c$ have opposite signs. We consider the case when they have the same sign first.

If $a$ and $c$ have the same sign, $g^{\prime}(x)$ will have no real roots and thus $g$ has no local extrema. Thus, in addition to the strip described above, quadrants I ( $a, c \geq 0)$ and III ( $a, c \leq 0)$ are part of the admissible region; see Figure 4, left.

If $a$ and $c$ have opposite signs, $g$ has local extrema. The roots of $g^{\prime}$ are outside the box if $-c / 3 a>1$. This corresponds to the cones bounded by $c= \pm 3 a$ in the $a c$ plane; see Figure 4, right.

On the other hand, if $-c / 3 a \leq 1$, or $|c| \leq 3|a|$, then $|g( \pm \sqrt{-c / 3 a})| \leq 1$ must be true. Evaluation at the critical points requires that

$$
-1 \leq g( \pm \sqrt{-c / 3 a}) \leq 1
$$

Without loss of generality, consider the case of $c$ negative. Now we are considering

$$
\begin{array}{lll}
-1 \leq a\left(\sqrt{\frac{-c}{3 a}}\right)^{3}+c \sqrt{\frac{-c}{3 a}} \leq 1 \quad & \Rightarrow & -1 \leq a\left(\frac{-c}{3 a}\right)^{3 / 2}+c \sqrt{\frac{-c}{3 a}} \leq 1 \quad \Rightarrow \\
-1 \leq a \frac{\sqrt{-c}}{3 a} \sqrt{\frac{-c}{3 a}}+c \sqrt{\frac{-c}{3 a}} \leq 1 \quad \Rightarrow \quad-1 \leq \frac{1}{3}|c| \frac{\sqrt{-c}}{\sqrt{3 a}}+c \frac{\sqrt{-c}}{\sqrt{3 a}} \leq 1 \quad \Rightarrow \\
-\sqrt{3 a} \leq \sqrt{-c}\left(-\frac{1}{3} c+c\right) \leq \sqrt{3 a} \quad \Rightarrow \quad-\sqrt{3 a} \leq \frac{2}{3} c \sqrt{-c} \leq \sqrt{3 a} .
\end{array}
$$

Next, we use this relation to solve for $c$ :

$$
\begin{aligned}
& -\sqrt{3 a} \leq \frac{2}{3}(-c)^{3 / 2} \leq \sqrt{3 a} \Rightarrow-\frac{3}{2} \sqrt{3 a} \leq(-c)^{3 / 2} \leq \frac{3}{2} \sqrt{3 a} \Rightarrow \\
& -c \leq\left(\frac{3}{2} \sqrt{3 a}\right)^{2 / 3} \Rightarrow-c \leq 3\left(\frac{1}{4}\right)^{1 / 3}(\sqrt{a})^{2 / 3} \Rightarrow c \geq-3(a / 4)^{1 / 3} .
\end{aligned}
$$




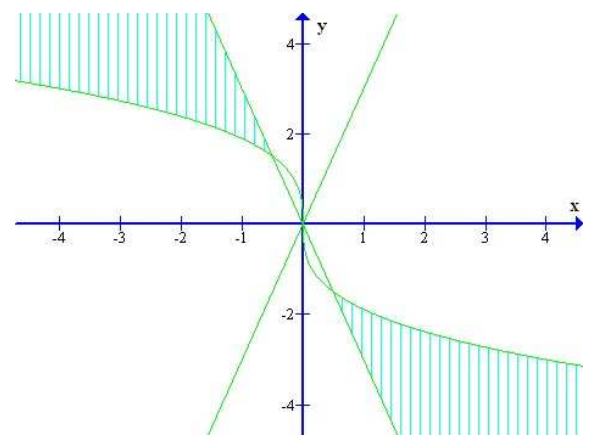

Figure 5. Cubic with cones, $a$ and $c$ of opposite signs, $|c| \leq 3|a|$. The vertical shading indicates regions of inadmissibility.

Similarly, when $c \geq 0$, we have

$$
c \leq 3(-a / 4)^{1 / 3} \text {. }
$$

The corresponding region is shown in Figure 5. Keep in mind that the cubic is relevant only outside the cones.

The combinations of the previous four regions is seen in Figure 6, which represents the total admissible area for $g(x)$.

Lastly, we consider the graph of $c$ in terms of $a$ from the condition to be an isometry (5-1). Graphing both the positive and negative roots reveals an ellipse, seen in Figure 7. This ellipse represents all the possibilities of $g(x)$ within the family. The parts that lie in the admissible region are shown with heavy printing. The positive and negative Chebyshev polynomials are at the extreme ends the major axis of the ellipse, sitting as isolated points. This means there exists a nontrivial family of isometries acting from $T_{1}$ to $T_{3}$, represented by the continuous arc of the ellipse in the admissible region, along with the isometries identified by Theorem 5 , represented by isolated points at the major vertices of the ellipse. Not only have these been identified, but these are the totality of all possible composition isometries between these subspaces. This is a very satisfying answer to the question.

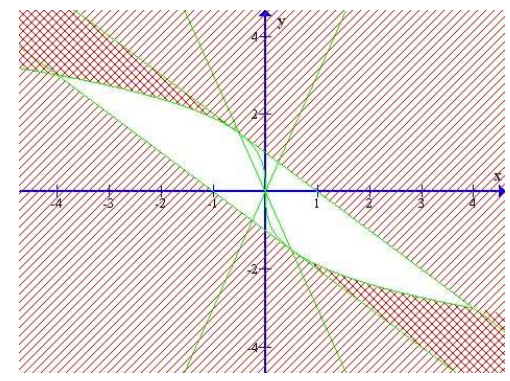

Figure 6. Total region of admissibility for $g(x)$ (unshaded). 


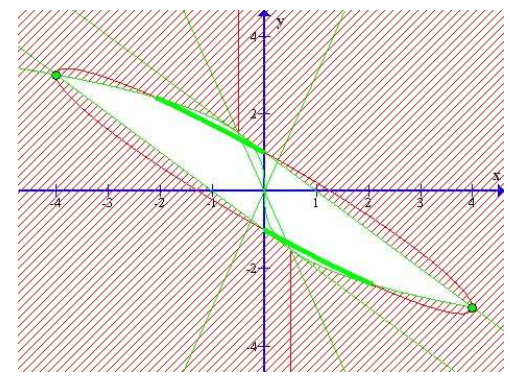

Figure 7. Admissible isometry-inducing symbols.

\section{Conclusion}

Some simple formulas for computing norms of composition operators on finitedimensional subspaces of the Chebyshev space show some direction for future lines of investigation. We raised the more specific question of isometric composition operators, especially in the case of $T_{1} \rightarrow T_{3}$. This revealed a family of operators whose symbols' coefficients vary over a continuum and a pair of isolated symbols corresponding to the Chebyshev polynomials of order 3 . The geometric connection to a norm question was surprising and pleasing.

\section{References}

[Akhiezer and Glazman 1993] N. I. Akhiezer and I. M. Glazman, Theory of linear operators in Hilbert space, Dover, New York, 1993. MR 94i:47001 Zbl 0874.47001

[Cowen and MacCluer 1995] C. C. Cowen and B. D. MacCluer, Composition operators on spaces of analytic functions, CRC Press, Boca Raton, FL, 1995. MR 97i:47056 Zbl 0873.47017

[Doperak 2006] J. Doperak, Norms of composition operators on the space of hermite polynomials, Summer Fellows undergraduate thesis, Ursinus College, 2006.

[Dunford and Schwartz 1958] N. Dunford and J. T. Schwartz, Linear operators, vol. 1, Wiley, New York, 1958. MR 90g:47001a Zbl 0084.10402

[Gareau 2005] M. Gareau, Composition operators acting on real-valued functions, Summer Fellows undergraduate thesis, Ursinus College, 2005.

[Horn and Johnson 1985] R. A. Horn and C. R. Johnson, Matrix analysis, Cambridge University Press, Cambridge, 1985. MR 87e:15001 Zbl 0576.15001

[Kunaszuk 2006] J. Kunaszuk, Norms of composition operators acting on the legendre polynomials, Summer Fellows undergraduate thesis, Ursinus College, 2006.

[Lebedev 1972] N. N. Lebedev, Special functions and their applications, revised ed., Dover, New York, 1972. MR 50 \#2568 Zbl 0271.33001

[Potter 2007] A. Potter, Composition operators acting on the Chebyshev space, Summer Fellows undergraduate thesis, Ursinus College, 2007.

[Reed and Simon 1980] M. Reed and B. Simon, Methods of modern mathematical physics, I: Functional analysis, 2nd ed., Academic Press, New York, 1980. MR 85e:46002 Zbl 0459.46001 
Received: 2008-11-10

tgoebeler@ursinus.edu

ashpot17@gmail.com
Revised: 2009-06-25 Accepted: 2009-08-12

Department of Mathematics and Computer Science, Ursinus College, Collegeville, PA 19426, United States

Department of Mathematics, Perkiomen Valley High School, 509 Gravel Pike, Collegeville, PA 19426, United States 